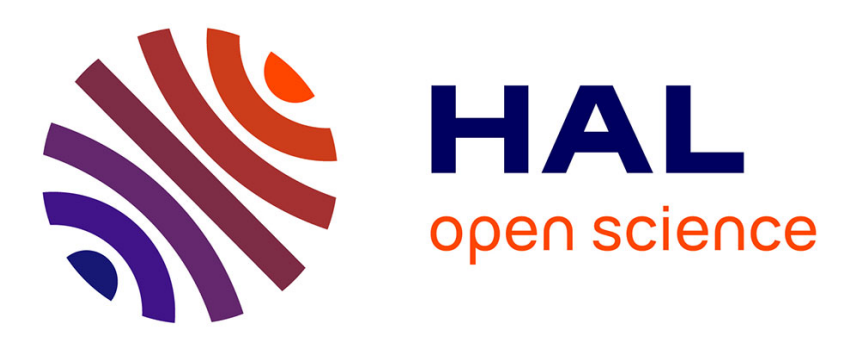

\title{
BOUNDED BEAM REFLECTION AND TRANSMISSION EFFECTS IN TERMS OF COMPLEX HARMONIC WAVES
}

K. van den Abeele, O. Leroy

\section{- To cite this version:}

K. van den Abeele, O. Leroy. BOUNDED BEAM REFLECTION AND TRANSMISSION EFFECTS IN TERMS OF COMPLEX HARMONIC WAVES. Journal de Physique IV Proceedings, 1992, 02 (C1), pp.C1-675-C1-678. 10.1051/jp4:19921146 . jpa-00251104

HAL Id: jpa-00251104 https://hal.science/jpa-00251104

Submitted on 1 Jan 1992

HAL is a multi-disciplinary open access archive for the deposit and dissemination of scientific research documents, whether they are published or not. The documents may come from teaching and research institutions in France or abroad, or from public or private research centers.
L'archive ouverte pluridisciplinaire $\mathbf{H A L}$, est destinée au dépôt et à la diffusion de documents scientifiques de niveau recherche, publiés ou non, émanant des établissements d'enseignement et de recherche français ou étrangers, des laboratoires publics ou privés. 


\title{
BOUNDED BEAM REFLECTION AND TRANSMISSION EFFECTS IN TERMS OF COMPLEX HARMONIC WAVES
}

\author{
K. VAN DEN ABEELE(1) and O. LEROY \\ Interdisciplinar Research Center, K.U.L. Campus Kortrijk, B-8500 Kortrijk, Belgium
}

\begin{abstract}
Surface waves, which are locally a combination of inhomogeneous waves, are found as an immediate consequence of the bounded beam representation in terms of complex harmonic waves. Decay and velocity of these waves can be obtained directly from the separation of the reflected field into an attendant reflected beam and an interface wave component. The influence of the bearnwidth parameter of the bounded beam on the decomposition into complex harmonic waves explains the nonobservance of particular Lamb waves at certain angular regions, e.g. near the longitudinal critical angle. This representation holds the possibility to predict optimum parameters to observe strong Lamb wave radiation which can be of great importance in the field of acoustic microscopy.
\end{abstract}

Résumé: Les ondes de surface, qui s'écrivent locallement comme des combinaisons d'ondes inhomogènes, résultent immédiatement d'une représentation des ondes bornées par des ondes harmonic complexes. Si on sépare le champ réfléchi en deux parties comme un champ attendu et un champ d'interface on trouve exactement la vitesse et la décroissance exponentielle de l'onde de surface. L'influence de la largeur de l'onde ultrasonore sur la décomposition en ondes inhomogènes explique le fait que l'onde de Lamb prévue pour certains angles, par exemple près de l'angle critique longitudinal ne se voit prèsque pas. Aves cette représentation nous avons la possibilité de calculer les paramètres de l'onde incidente pour qu'elle génère des fortes ondes de Lamb dont l'importance n'est pas négligeable en microscopy acoustique.

Apart from the fact that Fourier analysis has proven its applicability and dignity in a large number of fields 12 , it is sometimes difficult to account for the real physical phenomena happening behind the theoretical calculations. The generation of surface waves, e.g., explained in the classical way as a temporary conversion of a homogeneous wave into a surface wave which propagates over a certain distance along the interface and then reradiates by conversion into a heterogeneous bulk wave, is hard to understand. Furthermore, numerical problems arise in the description of the reflection and transmission of small bounded beams at large incidence angles.

The finite decomposition method of Claeys et al. ${ }^{3}$ interprets bounded beam deformations due to scattering at layered media with plane interfaces in terms of the reflection and transmission coefficients for bulk heterogeneous waves. Recent findings of Van Den Abeele et al. ${ }^{4}$ showed the existence of surface waves as part of the scattered field. In contrast with the classical ray formalism explaining the generation of surface waves, no propagation nor reradiation is involved in this model. We will first recall this method briefly and illustrate its ability to describe non-specular effects in reflection and transmission and even at large angles of incidence.

\section{Finite decomposition of bounded beams in terms of complex harmonic waves.}

Claeys et al. ${ }^{3}$ showed that it is possible to decompose any given profile into a set of heterogeneous waves satisfying the same dispersion relation. For a gaussian profile, e.g., it is sufficient to find the best set of amplitudes $A_{n}$ and heterogeneities $\beta_{n}$ for the complex harmonic waves for which $\sum_{n}^{N} A_{n} e^{\beta_{n} x_{i}}$ leads to the closest approximation of $\exp \left\{-\left(\mathrm{x}_{\mathrm{i}} / \mathrm{W}\right)^{2}\right\}$, where $\mathrm{x}_{\mathrm{i}}$ is the coordinate perpendicular to the direction of propagation and $W$ is the beamwidth parameter. A fairly good approximation was obtained 4 for $N=39, \beta_{n}=\frac{n}{1.9} W$ and $A_{n}$ (=A-n), independent of the width, given in Table 1. Each heterogeneous wave in this decomposition has the same direction

(1) Aspirant of the Belgian National Foundation for Scientific Research 
Table 1: Relative amplitudes $A_{n}$ of the heterogeneous waves used in the decomposition of a gaussian profile.

\begin{tabular}{|c|c|c|c|c|c|}
\hline $0-0.101585 \mathrm{E} 00$ & $0.623143 \mathrm{E} 02$ & $14-0.114722 \mathrm{E}-02$ & $210.428722 \mathrm{E}-11$ & $28-0.127424 \mathrm{E}-22$ & $\begin{array}{ll}35 & 0.136344 \mathrm{E}-37\end{array}$ \\
\hline $10.219627 \mathrm{E} 01$ & $8-0.253160 \mathrm{E} 02$ & $15 \quad 0.114003 \mathrm{E}-03$ & $22-0.148771 \mathrm{E}-12$ & $29 \quad 0.158749 \mathrm{E}-24$ & $36-0.405503 \mathrm{E}-40$ \\
\hline $2-0.209847 \mathrm{E} 02$ & $90.801411 \mathrm{E} 01$ & $16-0.963892 \mathrm{E}-05$ & $23 \quad 0.447807 \mathrm{E}-14$ & $30-0.168354 \mathrm{E}-26$ & $37 \quad 0.857345 \mathrm{E}$ \\
\hline $\begin{array}{ll}3 & 0.775777 \mathrm{E} 02\end{array}$ & $10-0.202240 \mathrm{E} 01$ & $17 \quad 0.696972 \mathrm{E}-06$ & $24-0.116916 \mathrm{E}-15$ & $31 \quad 0.150771 E-28$ & $38-0.11$ \\
\hline $4-0.141464 \mathrm{E} 03$ & $110.414019 \mathrm{E} 00$ & $18-0.432819 \mathrm{E}-07$ & $25 \quad 0.264569 \mathrm{E}-17$ & $32-0.112837 \mathrm{E}-30$ & $39 \quad 0.729231 \mathrm{E}-$ \\
\hline$\Rightarrow 5 \quad 0.155761 \mathrm{E} 03$ & $12-0.697106 \mathrm{E}-01$ & $19 \quad 0.231621 \mathrm{E}-08$ & $26-0.518114 \mathrm{E}-19$ & $33 \quad 0.695937 \mathrm{E}-33$ & \\
\hline $6-0.115878 \mathrm{E} 03$ & $13 \quad 0.976173 \mathrm{E}-02$ & $20-0.107098 \mathrm{E}-09$ & $27 \quad 0.875971 \mathrm{E}-21$ & $34-0.347116 \mathrm{E}-35$ & \\
\hline
\end{tabular}

of propagation as the approximated gaussian beam, defined by $\theta$, however, because of the difference in heterogeneity $\beta_{\mathfrak{n}}$ the value of the wavevector projection on an axis parallel to the interfaces ( $x$-axis) differs for each of

them : $\left[k_{n}\right]_{x}=k_{n}^{\prime} \sin \theta+i a_{n} \sin \theta-i \beta_{n} \cos \theta$

with $\mathrm{k}_{\mathrm{n}}^{\prime 2} \mathrm{a}_{\mathrm{n}}^{2}-\beta_{\mathrm{n}}^{2}=(\omega / \mathrm{v})^{2}\left(1-\left(\frac{\alpha}{2 \pi}\right)^{2}\right)$ and $\mathrm{k}_{\mathrm{n}}^{\prime} \cdot \mathrm{a}_{\mathrm{n}}=(\omega / \mathrm{v})^{2} \frac{\alpha}{2 \pi} \quad(\omega$ is the circular frequency), and $v$ and $\alpha$ are two medium constants : the velocity and the attenuation per unit of wavelength.

\section{Scattering of a bounded beam from a set of plane interfaces.}

In order to describe the scattered field of the gaussian beam at an arbitrary layered medium, we followed the following procedure. For each complex harmonic wave, the generalized laws of Snell-Descartes in combination with the appropriate dispersion relation determine the nature of all waves generated in the different layers and their directions with respect to the $z$-axis ${ }^{5}$. When these values are known, Brekhovskikh's theory, applied for complex wavenumbers and projections $\left[k_{n}\right]_{x}$, provides the reflection $\left(R\left(\left[k_{n}\right]_{x}\right)\right)$ and transmission $\left(T\left(\left[k_{n}\right]_{x}\right)\right)$ coefficients for the scattering of a plane acoustic heterogeneous wave incident on the considered layered sample. An example illustrating the behaviour of the modulus of the reflection coefficient as function of angle of incidence and heterogeneity is shown in Figure 2 (brass plate in water $f d=2.5$ ). The poles of $R$ (and $T$ ) correspond to the $x$ components of the leaky Lamb mode wavevectors. If $\mathbf{k}_{x}^{L}$ is such a root, the exact Lamb angle $\theta_{L}$, phase velocity $\omega / \mathrm{k}_{\mathrm{L}}$, attenuation $\mathrm{a}_{\mathrm{L}}$ and heterogeneity $\beta_{\mathrm{L}}$ of the corresponding leaky Lamb wave components in the liquid (with medium constants $v$ and $\alpha$ ) at both sides of the plate are found by solving following set of equations:

$$
k_{L} \sin \theta_{L}=\operatorname{Re}\left(k_{x}^{L}\right) ; a_{L} \sin \theta_{L}-\beta_{L} \cos \theta_{L}=\operatorname{Im}\left(k_{x}^{L}\right) ; k_{L}^{2}-a_{L}^{2}-\beta_{L}^{2}=(\omega / v)^{2}\left(1-\left(\frac{\alpha}{2 \pi}\right)^{2}\right) ; k_{L} \cdot a_{L}=(\omega / v)^{2} \frac{\alpha}{2 \pi} .
$$

(Remark that, because $\beta_{L}$ is different from zero, this solution is somewhat different from the calculations usually found in literature ${ }^{1}$.)

Such solution $\left(k_{L}, a_{L}, \beta_{L}, \theta_{L}\right)$ with $0 \leq \theta_{L}<\pi / 2$ has to be interpreted as the particular incident heterogeneous wave for which a leaky Lamb wave is generated inside the plate travelling with velocity $\omega / \operatorname{Re}\left(k_{L}\right)$ along the $x$-direction while energy is leaking through the interfaces above and underneath the plate. These energy flows are represented by two heterogeneous waves: $\left(k_{L}, a_{L},-\beta_{L}, \pi-\theta_{L}\right)$ in reflection and $\left(k_{L}, a_{L}, \beta_{L}, \theta_{L}\right)$ in transmission. The characteristics of the wave mode inside the plate immediately follow from the generalized laws of Snell-Descartes.

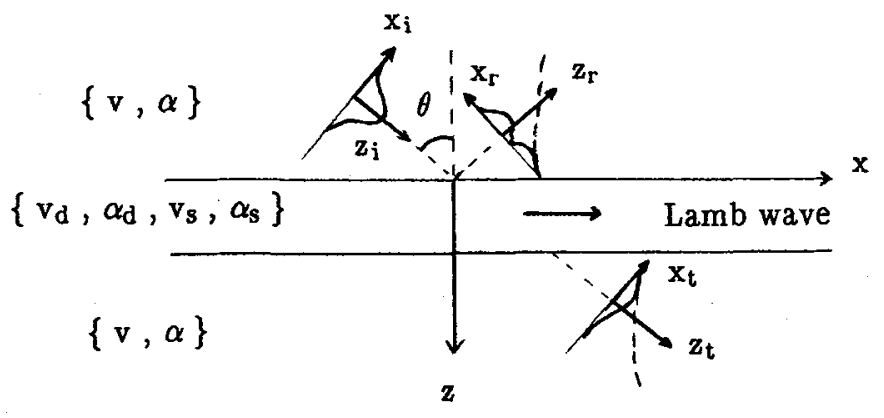

Figure 1: Geometry of the bounded beam reflection and transmission. 


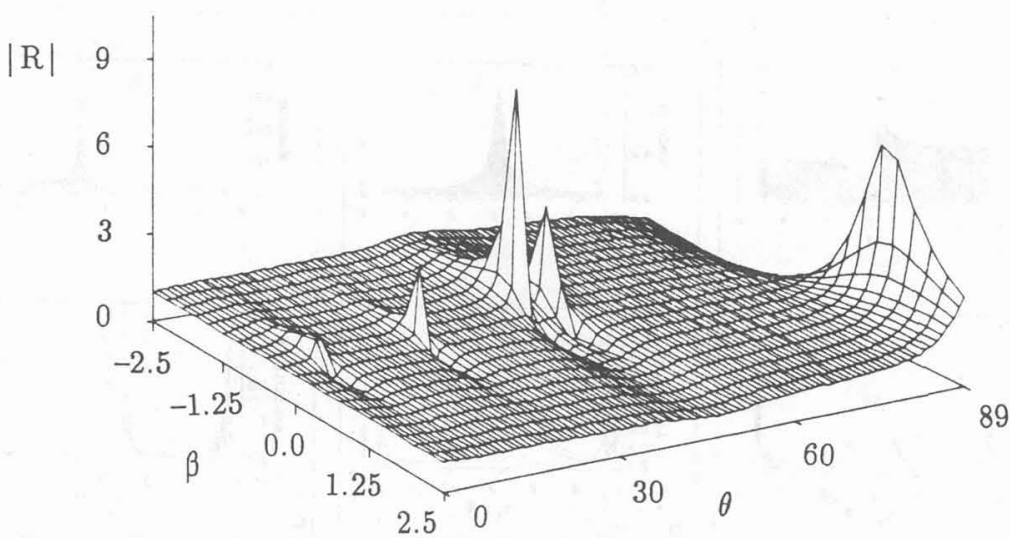

Figure 2: Reflectioncoefficient of complex harmonic waves as function of incidence angle and heterogeneity for a brass plate of $0.5 \mathrm{~mm}$ in water (frequency $f=5 \mathrm{MHz}$ ) with small absorption.

When using an appropriate grid, the threedimensional surface representation of $|\mathrm{R}|$ as function of $\beta$ and $\theta$ immediately yields all possible combinations leading to the excitation of plate vibrations and for $\theta \rightarrow 90^{\circ}$ even the Stoneley wave can be found. It is important to note that no temporarely propagation along the surface and no re-emission is assumed. The excitation of surface waves can be explained as a pure reflection/transmission phenomenon of heterogeneous waves.

Because of linearity, the amplitude and phase distribution of the reflected and transmitted profile for any angle of incidence $\theta$ can be found by multiplying each heterogeneous wave by its complex valued reflection or transmission coefficient. Van Den Abeele et al. ${ }^{4}$ showed that, for instance for reflection, the scattered field

$\stackrel{3}{\Sigma}^{39} \mathrm{R}\left(\left(\mathrm{k}_{\mathrm{x}}\right)_{\mathrm{n}}\right) \mathrm{A}_{\mathrm{n}} \mathrm{e}^{-\beta_{\mathrm{n}} \mathrm{x}_{\mathrm{r}}}$ at $z_{\mathrm{r}}=0$ can be separated with significant physical meaning into two components. If $\mathrm{n}=-39$

$\theta$ is close to a Lamb angle corresponding to the solution $\left(k_{L}, a_{L}, \beta_{L}, \theta_{L}\right)$ with $\beta_{L}<0$, the first one is mathematically

described by $R\left(\left(k_{x}\right)_{0}\right) A_{0}+2 \sum_{n=1}^{39} R\left(\left(k_{x}\right)_{n}\right) A_{n} e^{-\beta_{n} x_{I}}$

and represents the attendant reflected profile (same result as Fourier analysis predicts) while the second component is highly connected to the energy of the plate mode leaking into the liquid:

$$
\sum_{n=1}^{39} A_{n}\left\{R\left(\left(k_{x}\right)_{-n}\right) e^{\beta_{n} x_{r}}-R\left(\left(k_{x}\right)_{n}\right) e^{-\beta_{n} x_{r}}\right\}
$$

For transmission one can follow exactly the same procedure. For angles close to a Lamb angle for which $\beta_{\mathrm{L}}>0$ in the solution $\left(k_{L}, a_{L}, \beta_{L}, \theta_{L}\right)$ and for the Stoneley solution $\left(\beta_{S}>0 \text { and } \theta_{S}=90^{\circ} \text { i.e. grazing incidence !!! }\right)_{1}$ the summation for the index $\mathrm{n}$ in both components has to extend from -39 up to -1 instead of summing from 1 to 39 . In Figure 3a-3c some results for non-specular gaussian beam reflection and transmission are illustrated. Every illustration is accompanied by a figure showing the variation of the reflection/transmission coefficient for the discrete heterogeneous waves which build up the incident profile.

A very interesting study concerns the influence of beamwidth variations of the incident gaussian beam on the excitation of leaky Lamb wave fields at fixed angles of incidence. Since $\mathrm{A}_{5}$ is the largest among the coefficients in the decomposition of the incident beam, the contribution of the leaking energy flow component reaches its maximum value if the heterogeneous wave with this amplitude has the largest reflection coefficient among all, i.e. if $\left|\beta_{5}\right|=\left|\beta_{L}\right|$. This means that the optimum beamwidth in order to generate strong leaky Lamb waves at $\theta_{L}$ corresponds to a width of $W \approx\left[0.38\left|\beta_{L}\right|\right]^{-1}$. If the beam is too wide, the heterogeneous wave with the peculiar heterogeneity for which high values of the reflection coefficient occur is located too far from the region of waves with significant amplitudes $A_{n}$, which results in the nonobservance of any leaking energy from a surface wave. Nevertheless, the profile may be reduced in amplitude because of a global reflection coefficient smaller than one for all heterogeneous waves in (1) (Figure 3d). However if the beamwidth is too small, it may also be impossible to obtain energy radiation of a leaky Lamb wave into the liquid. This explains the nonobservance of nonspecular reflectivity at Lamb angles corresponding to symmetric lamb modes in the neighbourhood of the longitudinal 

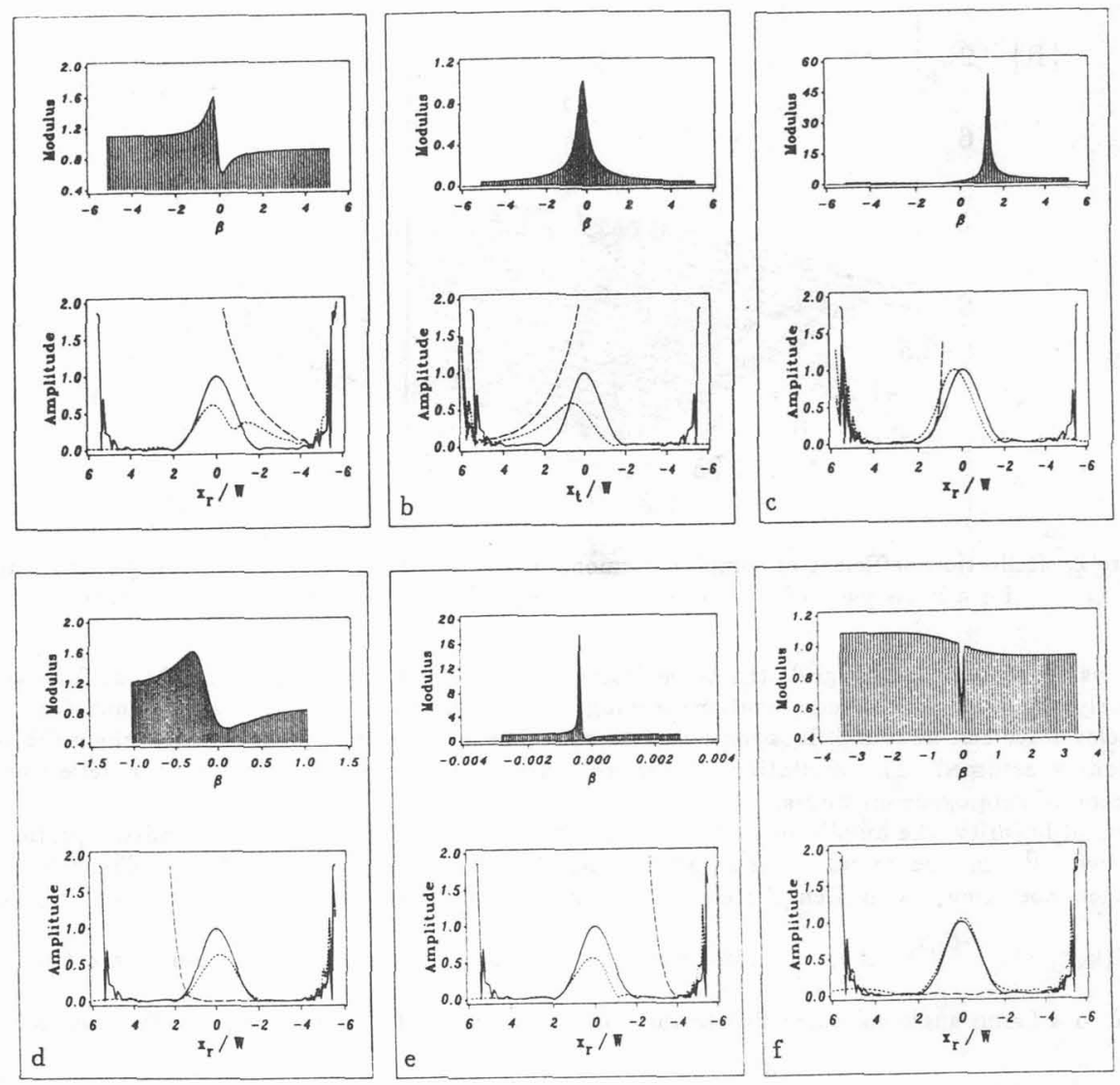

Figure 3: Bounded beam deformations (short dashed line) and leaky surface wave components (long dashed line) as a result of the scattering of a gaussian beam incident on a brass plate of $0.5 \mathrm{~mm}$ at $5 \mathrm{MHz}$ with small absorption. The incident profile, as a reference, is given by the full line.

$3 \mathrm{a}$ and $\mathrm{b}:$ Lamb wave influence on reflection and transmission at $\theta=24.5^{\circ}, \mathrm{W}=4 \mathrm{~mm}$.

$3 \mathrm{c}$ : Stoneley wave influence on reflection at $\theta=89.9^{\circ}, \mathrm{W}=4 \mathrm{~mm}$

$3 \mathrm{~d}$ : Lamb wave influence on reflection at $\theta=24.5^{\circ}$, for increased beamwidth $W=20 \mathrm{~mm}$.

3e and $\mathrm{f}: \theta=19.76228^{\circ} \approx \theta_{\text {lc }}$, for optimum beamwidth $\mathrm{W}=7310 \mathrm{~mm}$ and experimentally ${ }^{6}$ used beamwidth $W=6 \mathrm{~mm}$.

critical angle $\theta_{1 \mathrm{c}}$ which has been reported by de Billy et al. ${ }^{6}$. Indeed, by choosing the grid used in figure 2 more appropriate it is possible to detect a "hidden" pole of the reflection coefficient corresponding to the $\mathrm{S}_{1}$ mode near

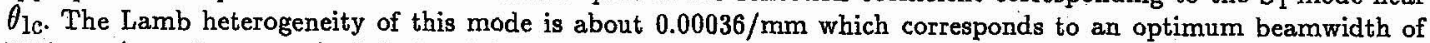
$7310 \mathrm{~mm}$ ( $\approx$ a plane wave). Calculated deformations for this beamwidth are given in Figure 3e. The reason why de Billy et al. observed only some weak radiation is thus simply due to use of a transducer with dimensions which are too small (Figure 3f). For less extreme cases (e.g. for mode $A_{1}: \theta=24.02^{\circ}, W_{\text {opt }}=15.04 \mathrm{~mm}$ ), the optimum beamwidth determination can be of great inportance in acoustic microscopy e.g. to adjust the dimensions of a gaussian profile in order to obtain in any situation the strongest possible leaky wave energy flow.

\section{References:}

/1/ T.J. Plona, L.E. Pitts and W.G. Mayer, J.A.S.A. $\underline{59}$ (6), 1324-1328 (1976).

/2/ T.D.K. Ngoc and W.G. Mayer, IEEE Trans. on Sonics and Ultrasonics, SU 27 (5), $229-236$ (1980).

/3/ J.M. Claeys and O. Leroy, J.A.S.A. 72 (2), 585-590 (1982).

/4/ K. Van Den Abeele and O. Leroy, submitted to J.A.S.A.

/5/ M. Deschamps, J. Acoustique 4, 269-305 (1991) and references therein.

/6/ M. de Billy and I. Molinero, J.A.S.A. 83 (4), 1249-1254 (1988). 